\title{
Synergetic Effect of In and Ag Co-doped ZnS for Enhanced Photocatalytic Hydrogen Evolution under Visible Light Irradiation
}

\author{
Melody Kimi ${ }^{1,2, a^{*}}$, L. Yuliati ${ }^{3, b}$, M. Shamsuddin ${ }^{1, c}$ \\ ${ }^{1}$ Department of Chemistry, Faculty of Science, Universiti Teknologi Malaysia, 81310 Johor Bahru, \\ Johor, Malaysia. \\ ${ }^{2}$ Centre for Pre-University Studies, Universiti Malaysia Sarawak, 94300 Kota Samarahan, \\ Sarawak, Malaysia.
}

${ }^{3}$ Ibnu Sina Institute for Fundamental Science Studies, Universiti Teknologi Malaysia, 81310 Johor

Bahru, Johor, Malaysia.

akmelody@preuni.unimas.my, ${ }^{\mathrm{b}}$ leny@ibnusina.utm.my, ${ }^{\mathrm{c}} \mathrm{mustaffa@kimia.fs.utm.my}$

Keywords: hydrogen, visible light, ZnS, co-doped, photocatalyst

\begin{abstract}
In (Indium) and Ag (Argentum) co-doped $\mathrm{ZnS}$ photocatalysts were successfully prepared by hydrothermal method to extend the light absorption of $\mathrm{ZnS}$ to the visible light region. The concentration of In was constant while for Ag was varied to optimize the photocatalytic activity. The In and Ag co-doped ZnS (Zinc Sulfide) photocatalysts showed smaller band gap energy compared to single doped $\operatorname{In}(0.1)-\mathrm{ZnS}$ and undoped $\mathrm{ZnS}$. The photocatalytic activity of In and $\mathrm{Ag}$ co-doped $\mathrm{ZnS}$ photocatalysts was evaluated from the amount of hydrogen produced. The hydrogen evolution rate from aqueous solution containing $\mathrm{Na}_{2} \mathrm{SO}_{3}$ (Sodium Sulfite) and $\mathrm{Na}_{2} \mathrm{~S}$ (Sodium Sulfide) as sacrificial reagent under visible light irradiation obtained from In and Ag co-doped $\mathrm{ZnS}$ is higher compared to the single doped $\operatorname{In}(0.1)-\mathrm{ZnS}$ when optimum amount of Ag dopant was added. The highest photocatalytic activity is observed for $\operatorname{In}(0.1), \operatorname{Ag}(0.01)-\mathrm{ZnS}$ with hydrogen production rate of $26.82 \mu \mathrm{mol} / \mathrm{h}$. The higher performance of this photocatalyst is ascribed to the extended visible light absorption, efficient charge separation as well as improved electron transfer associated with synergistic effect of appropriate amount of In and Ag co-doped $\mathrm{ZnS}$.
\end{abstract}

\section{Introduction}

Hydrogen production under solar light irradiation with the assistance of semiconductor photocatalyst is one of the potential solutions to resolve the depletion of fossil fuels [1]. $\mathrm{ZnS}$ possesses high conduction band that is essential for photocatalytic hydrogen production [1] but ZnS is not active under visible light irradiation. Indium based sulfide material has gain interest due to the visible light active properties which can utilize most of the solar light energy. The $\operatorname{In}_{2} \mathrm{~S}_{3}$ (Indium Sulfide) has showed good efficiency for hydrogen production under visible light irradiation [2]. Thus, indium can be doped into $\mathrm{ZnS}$ to improve the absorption properties. Ag dopant is frequently used as electron trap, enhancing electron-hole separation and promoting the interfacial electron transfer processes [3]. The addition of Ag increases the ability of a photocatalyst to absorb visible light, due to localized surface Plasmon resonance [4]. Studies on Ag doped ZnS for hydrogen production has also been done previously [5]. It would be beneficial if both In and Ag are doped into $\mathrm{ZnS}$ to improve the visible light absorption and also enhance the photocatalytic activity by inhibiting fast electron-hole recombination. In this study, $\operatorname{In}(0.1), \operatorname{Ag}(x)-\mathrm{ZnS}$ was successfully synthesized by hydrothermal method. The amount of Ag was varied to optimize the performance of the photocatalysts and systematically studied the physical-chemical properties and photocatalytic activities under visible light irradiation. The effects of co-doping on the photocatalytic activities were also discussed. 